\title{
Nonadiabatic Excited-State Molecular Dynamics: On-the-Fly Limiting of Essential Excited States
}

\author{
Tammie Nelson ${ }^{\mathrm{a}}$, Artem Naumov ${ }^{\mathrm{b}}$, Sebastian Fernandez-Alberti ${ }^{\mathrm{c}}$, Sergei \\ Tretiak $^{\mathrm{a}, *}$ \\ ${ }^{a}$ Los Alamos National Laboratory, Los Alamos, New Mexico 87545, USA \\ ${ }^{b}$ Skolkovo Institute of Science and Technology, Moscow 143026, Russia \\ ${ }^{c}$ Universidad Nacional de Quilmes, Roque Saenz Pea 352, B1876BXD Bernal, Argentina
}

\begin{abstract}
The simulation of nonadiabatic dynamics in extended molecular systems involving hundreds of atoms and large densities of states is particularly challenging. Nonadiabatic coupling terms (NACTs) represent a significant numerical bottleneck in surface hopping approaches. Rather than using unreliable NACT cutting schemes, here we develop "on-the-fly" state limiting methods to eliminate states that are no longer essential for the non-radiative relaxation dynamics as a trajectory proceeds. We propose a state number criteria and an energy-based state limit. The latter is more physically relevant by requiring a user-imposed energy threshold. For this purpose, we introduce a local kinetic energy gauge by summing contributions from atoms within the spatial localization of the electronic wavefunction to define the energy available for upward hops. The proposed state limiting schemes are implemented within the nonadiabatic excited-state molecular dynamics framework to simulate photoinduced relaxation in poly-phenylene vinylene (PPV) and branched poly-phenylene ethynylene (PPE) oligomers for benchmark evaluation.
\end{abstract}

Keywords: fewest switches surface hopping; NA-ESMD; excited state limiting; local kinetic energy; poly-phenylene vinylene; poly-phenylene ethynylene

\section{Introduction}

The computational simulation of photochemical and photophysical processes governed by nonadiabatic (NA) dynamics in polyatomic molecules is one of the main driving forces in the field of molecular excitonics. NA dynamics is respon-

5 sible for many fundamental photophysical processes, such as charge and energy transfer, that can be modeled using direct nonadiabatic molecular dynamics (NA-MD). Molecular dynamics with quantum transitions (MDQT) based on Tully's fewest-switches surface hopping (FSSH) algorithm [1] is a computationally reliable and robust method for performing NA-MD simulations in large

*serg@lanl.gov

Preprint submitted to Chemical Physics

May 16, 2016 
systems. It involves the separate treatment of electronic (quantum) and nuclear (classical) subsystems by propagating nuclear trajectories on an electronic potential energy surface (PES) defined by a single adiabatic state and allowing transitions between coupled electronic states based on the nonadiabatic coupling strengths. The popularity of this method cannot be understated, and it 15 has been applied successfully to problems ranging from charge/energy transfer $[2,3,4,5,6]$ and proton transfer $[7,8,9]$ to collision reactions $[10,11]$ and biological systems. $[12,13]$

Over the years, we have developed a nonadiabatic excited-state molecular dynamics (NA-ESMD) framework using the FSSH algorithm to efficiently and

20 accurately model NA dynamics in extended molecular systems. [14, 15] Our methodology employs the Configuration Interaction Single (CIS) formalism [16] with a semiempirical Hamiltonian allowing a reasonable description of the multiple excited states to be achieved at a relatively low computational cost. By taking advantage of available analytical derivative techniques, $[17,18,19,20,21]$

25 NA-ESMD provides an ideal method for simulating photoinduced dynamics in systems with hundreds of atoms involving many ( 100) coupled excited states on time scales of up to $\sim 10$ ps.

Extended molecular systems and condensed phase systems are particularly challenging. These systems often contain hundreds of atoms and have large

30 densities of excited states participating in the NA dynamics making their simulation computationally demanding. Efficient computation of electronic energies, gradients, and nonadiabatic coupling terms (NACTs) is the primary bottleneck in this field of theoretical simulations. For a complete coupling model involving $\mathrm{N}_{S}$ electronic states, there are $\mathrm{N}_{S}\left(\mathrm{~N}_{S}-1\right) / 2$ non-redundant NACTs to be 35 computed. A common approximation introduced to alleviate the computational demand represented by the NACTs is to reduce the number of coupling terms included in the simulation. Over the years, various NACT cutting schemes have been proposed. [22, 23] However, these approximations quickly break down for large systems and are only valid for simulations involving a few $(2-3)$ ex-

40 cited states, making them impractical for condensed phase applications. [24] Furthermore, NACT cutting schemes are indiscriminate and introduce error in the propagation of the electronic wavefunction by truncating the summation of NACT contributions entering the equations of motion.

Here, we propose that system size constraints can instead be overcome by 45 introducing an "on the fly" limit for the number of essential excited states included in the simulation. At each hopping instance, states that are no longer relevant for the relaxation dynamics can be removed, thus the computational demand associated with evaluating NACTs and energies of many excited states will be reduced as the simulation progresses. As a prerequisite for such an 50 approach, instantaneous decoherence is used to reset the quantum amplitude of the current state after every attempted hop so that the system collapses to a pure state. [25] In this way, ambiguity in dealing with the redistribution of adiabatic state populations upon removal of states is avoided.

We introduce two possible methods for performing the state limitation: (1) ${ }_{55}$ The state number criteria related to the number of states above the current 
state that will be retained and (2) energy-based state limit in which only states within a defined energy window above the current occupied state are retained. While the state number criteria is arbitrary, an obvious choice for defining an energy window in energy-based state limit can be related to the kinetic energy.

60 Transitions to higher energy states are classically forbidden if there is insufficient kinetic energy (in the direction of the nonadiabatic coupling) to conserve the total energy. While the total kinetic energy represents an upper bound for the range of states that could be accessed by a hop to higher energy, in practice only the contribution to the kinetic energy from atoms within the spatial localiza-

65 tion of the electronic wavefunction should be available for hops to higher energy. This has been confirmed by several previous works analyzing the intramolecular vibrational energy flow accompanying electronic energy transfer. [26, 27] Therefore, we introduce a local kinetic energy to define the relevant energy window. Any state above that threshold would no longer be involved in the electronic 70 propagation.

Here, we implement our proposed "on the fly" state limiting schemes within the NA-ESMD framework by modeling the photoinduced dynamics in oligomer systems of poly-phenylene vinylene (PPV) and branched poly-phenylene ethynylene (PPE), two systems studied extensively by our group. [14, 5, 28, 24, 29,

$7525,6,26,30,31]$ We evaluate the accuracy of the proposed limitation criteria and discuss their advantages and limitations. We find that the surface hopping algorithm is robust to state limiting and directions for future improvement of computational efficiency have been identified.

\section{Theoretical Methodology}

\subsection{NA-ESMD}

NA-ESMD uses the FSSH algorithm combined with electronic energies, gradients, and nonadiabatic coupling vectors for the excited states calculated "on the fly" using the Collective Electronic Oscillator (CEO) method. [32, 33] The CEO method is applied at the Austin Model 1 (AM1) [34] level of theory using

85 the Configuration Interaction Singles (CIS) formalism [16] to describe correlated excited states. A detailed description of the NA-ESMD implementation is available elsewhere. [14, 15, 25, 24, 28]

In accordance with standard FSSH, [1] for each trajectory, the forces acting on the nuclei are determined by a single adiabatic state, the current state $\alpha$. The total electronic wavefunction is a mixed state given by

$$
\Psi(x, R, t)=\sum_{i} c_{i}(t) \phi_{i}(\mathbf{r} ; \mathbf{R}(\mathbf{t})),
$$

where $\phi_{i}(\mathbf{r} ; \mathbf{R}(\mathbf{t}))$ are the adiabatic basis functions with their respective timedependent expansion coefficients $c_{i}(t)$, and $\mathbf{r}$ represents the electronic degrees of freedom. The Schrödinger equation of motion for the coefficients is given by

$$
i \hbar \frac{\partial c_{i}(t)}{\partial t}=c_{i}(t) E_{i}-i \hbar \sum_{j} c_{j}(t) \dot{\mathbf{R}} \cdot \mathbf{d}_{\mathbf{i j}}
$$


where $\mathbf{d}_{\mathbf{i j}}=\left\langle\phi_{i}(R) \mid \nabla_{R} \phi_{j}(R)\right\rangle$ defines the nonadiabatic coupling vector and the

${ }_{95}$ product $\dot{\mathbf{R}} \cdot \mathbf{d}_{\mathbf{i j}}=\left\langle\phi_{i} \mid \frac{\partial \phi_{j}}{\partial t}\right\rangle$ is the scalar NACT used to determine hopping probabilities. Any instances of trivial unavoided crossings during the dynamics are treated using the previously developed Min-cost assignment algorithm. [28, 29] Instantaneous decoherence [25] is introduced in order to account for divergent wavepackets by reinitializing quantum coefficients after each attempted hop (both successful and forbidden). A coefficient of one is assigned to the current state and zero for all other states to collapse the wavefunction to a pure electronic state. Instantaneous decoherence is an ad hoc correction based on the assumption that hopping events can be used to signal nonadiabatic coupling regions and that wave packets evolving on different surfaces will instantly separate 105 in phase space. Despite these assumptions, the resetting of quantum coefficients has been shown to successfully eliminate internal inconsistency $[25,35,36]$ and it is an essential ingredient for the state limiting methods proposed here, as explained in the next section.

\subsection{Limiting Essential Excited States}

110

At every timestep for which a hop is attempted, the number of states to be carried on in the subsequent dynamics is evaluated. This is convenient because the wavefunction is collapsed at every attempted hop in the instantaneous decoherence implementation. [25] At the moment of hop, the current state will always be retained and it represents the entire instantaneous electronic wavepacket. This removes any concern about arbitrarily redistributing the quantum amplitude from states that may be removed.

We introduce two methods, outlined in Scheme 1, to define the state limit: the state number criteria and the energy-based state limit. In the state number criteria, states that are within a defined threshold, $n$, above the current state 120 are retained. For current state $\alpha$, any state above $S_{\alpha+n}$ is removed. For the energy-based state limit, an energy window, $\mathrm{E}_{\text {cut }}$, is defined and any states that are higher in energy than $\mathrm{E}_{\alpha}+\mathrm{E}_{\text {cut }}$ are removed. The threshold $\mathrm{E}_{c u t}$ should be chosen at each attempted hop based on the energy available for transitions to higher energy. Naturally, the total kinetic energy represents the largest possible

${ }_{125}$ value for this energy cutoff. Any states higher in energy will no longer be accessible during the dynamics and can be removed.

The photoinduced energy transfer represents a concerted electronic and vibrational process. [15] Ideally, only the kinetic energy contribution from atoms within the spatial localization region of the electronic wavefunction should con130 tribute to the energy available for hops to higher energy. This requirement becomes apparent when one considers a system involving spatially localized excitons in well separated molecular fragments. In order to calculate this local kinetic energy, the portion of atoms involved in the localization of the wavefunction must be determined. For that purpose, we rely on the electronic transition density (TD) and respective participation number per atom.

The electronic transition density represents the spatial localization of an excitation and can be used as a simplified measure of the electronic wavefunc- 
tion localization. Within the CEO approach, transition density matrices between the CIS adiabatic ground and excited state wave functions $\phi_{g}(r ; \mathbf{R}(t))$ and $\phi_{\alpha}(r ; \mathbf{R}(t))$, respectively, are given by

$$
\left(\rho^{g \alpha}\right)_{n m}=\left\langle\phi_{\alpha}(r ; \mathbf{R}(t))\left|c_{m}^{+} c_{n}\right| \phi_{g}(r ; \mathbf{R}(t))\right\rangle
$$

where $n$ and $m$ are atomic orbital indices and $c_{m}^{+}$and $c_{n}$ are creation/annihilation operators. The net electronic density change caused by $g \rightarrow \alpha$ excitation is given by the diagonal elements $\left(\rho^{g \alpha}\right)_{n n}$. In this way, the transition density localization of each atom (index A) in a system can be computed as $\left(\rho^{g \alpha}\right)_{n m}^{2}$. Next, we compute the participation number per atom

$$
P=\left[\sum_{A}\left(\sum_{n_{A}}\left(\rho^{g \alpha}\right)_{n_{A} n_{A}}^{2}\right)^{2}\right]^{-1}
$$

where the index $A$ runs over all atoms. The participation number per atom reveals the extent of localization/delocalization. For a system composed of $N$ atoms, the participation number can vary from 1 to $N$ where $P=1$ indicates complete localization of the electronic transition density within a single atom, and $P=N$ corresponds to complete delocalization with TD distributed over all $N$ atoms. The first $P$ atoms (ordered in decreasing value of their contribution to the total TD) retain the main spatial localization of the electronic excited state wavefunction. Therefore, only the kinetic energy for these $P$ atoms with largest transition density (index $B$ ) is summed

$$
K E_{T D}=\sum_{P} \frac{1}{2} m_{B} v_{B}^{2} .
$$

155 Here, $K E_{T D}$ represents the local kinetic energy contributed by the atoms where the excitation (wavefunction) is localized based on the TD analysis. This quantity can now be used to define a physically relevant energy window for the energy-based state limit.

\subsection{Molecular Dynamics Simulations}

We have performed NA-ESMD simulations of the photoinduced dynamics in the model poly-phenylene vinylene (PPV) and poly-phenylene ethynylene (PPE) oligomer systems depicted in Figure 1. The PPV3 system is composed of a 3-ring PPV oligomer and the 2-3-4 PPE system consists of linear 2-ring, 3ring, and 4-ring PPE units linked by meta-substitutions. Simulations are carried out at the AM1/CIS level of theory to model the room temperature (300 K) dynamics as the systems relax from a high energy excited electronic state back to the lowest energy $\mathrm{S}_{1}$ state.

Simulations are started by propagating a 600 ps ground-state (GS) BornOppenheimer trajectory using a timestep of $\Delta t=0.5 \mathrm{fs}$. During the first $10 \mathrm{ps}$, 170 the systems are heated to a temperature of $300 \mathrm{~K}$ maintained using the Langevin thermostat [37] with a friction coefficient $\gamma=2.0 \mathrm{ps}^{-1}$. Initial configurations 
and velocities were sampled from the equilibrated GS trajectory for the subsequent excited state simulations with each snapshot corresponding to an independent trajectory. For each sampled GS configuration, single point calculations were performed using the CEO method to obtain vertical excitation energies and oscillator strengths. The initial excited state was chosen according to a Franck-Condon window defined as

$$
g_{i}(r, \mathbf{R})=\exp \left[-T^{2}\left(E_{\text {laser }}-\Omega_{i}\right)^{2}\right]
$$

where $E_{\text {laser }}$ is the excitation energy and $\Omega_{i}$ is the calculated energy of state $i$ (units of $\mathrm{fs}^{-1}$ ). The simulated laser shape is Gaussian $f(t)=\exp \left(-t^{2} / 2 T^{2}\right)$ 180 with a FWHM of $100 \mathrm{fs}$. The relative values of $g_{i}(r, \mathbf{R})$ weighted by the oscillator strengths of each state $i$ are then used to select the initial state. The ensemble of excited state energies and oscillator strengths can be used to create an equilibrated absorption spectrum by making a histogram of the excited state energies where the height of the histogram is related to the average ratio between oscillator strength and frequencies for the corresponding state.

For PPV, GS snapshots were collected at 500 fs intervals. The well-known $m A_{g}\left(\mathrm{~S}_{m}\right)$ state appearing in the excited state absorption was chosen as the initial state. The $\mathrm{S}_{m}$ state corresponds to a delocalized excitonic state in conjugated polymers and is the state with the largest oscillator strength from $190 \mathrm{~S}_{1} \cdot[14,38,39]$ The electronic transition densities for the $\mathrm{S}_{1}$ and $\mathrm{S}_{m}$ states are shown in Figure 2, confirming that both states correspond to delocalized transitions. The equilibrated absorption spectrum and density of excited states (DOES) for PPV is shown in the top panel of Figure 3 where 15 lowest energy excited states are considered. Both the linear $S_{n} \leftarrow S_{0}$ (black) and nonlinear $\mathrm{S}_{n}$

${ }_{195} \leftarrow \mathrm{S}_{1}$ (red) spectra are shown where the largest contribution to the nonlinear spectrum corresponds to the $\mathrm{S}_{m}$ state. Excitation to $\mathrm{S}_{m}$ was performed according to Eq. 6 using a laser centered at $\lambda_{\text {laser }}=245 \mathrm{~nm}$ according to the calculated $\mathrm{S}_{n} \leftarrow \mathrm{S}_{1}$ absorption, shown in red in Figure 3. Due to conformational disorder at room temperature, typical values of $m$ vary from 8 to 12 (highlighted in blue in the DOES in Figure 3). A swarm of 540 NA-ESMD trajectories was propagated at $300 \mathrm{~K}$ for $1 \mathrm{ps}$ using a classical time step $\Delta t=0.1$ fs with $N_{q}=3$ quantum steps per classical step.

For PPE, GS snapshots were collected at 1 ps intervals. The meta-branching of the PPE system causes excitons to be localized within each linear PPE fragment as seen in the plots of the electronic transition density for the 4 lowest energy vertical excitations shown in Figure 2. The difference in length of the fragments creates an intramolecular energy gradient such that energy transfer occurs from the high energy 2-ring fragment to the lowest energy 4-ring fragment. Excitation to the state localized on the 2-ring fragment $\left(\mathrm{S}_{2-\text { rings }}\right)$ was performed according to Eq. 6 using a laser centered at $\lambda_{\text {laser }}=346 \mathrm{~nm}$. The equilibrated absorption spectrum and DOES for PPE is shown in the bottom panel of Figure 3 where 8 lowest energy excited states have been included. As can be seen, the absorbance of individual states strongly overlaps causing $\mathrm{S}_{2 \text {-rings }}$ to correspond to the range of states from $\mathrm{S}_{4}$ to $\mathrm{S}_{7}$ (highlighted in magenta in the 
DOES in Figure 3). A swarm of 490 NA-ESMD trajectories was propagated at $300 \mathrm{~K}$ for 200 fs using a classical time step $\Delta t=0.2$ fs with $N_{q}=4$ quantum steps per classical step.

\section{Results \& Discussion}

We start by verifying that our NA-ESMD simulations are amenable to state limiting. An important factor in this consideration is that the results of the full simulation without state limiting must be independent of the number of excited states included in the simulation, $\mathrm{N}_{S}$. This requirement ensures that no artifacts are introduced from varying the number of states during the course of the simulation with state limiting. In order to test this, room temperature NA-ESMD

225 simulations without state limiting were performed for PPV and PPE where $\mathrm{N}_{S}$ has been varied. For PPV, $\mathrm{N}_{S}$ was increased from 15 to 18, and finally 22 . For PPE, $\mathrm{N}_{S}$ values of 10,15 , and 20 were tested. The relaxation dynamics can be followed by analyzing the evolution of the adiabatic state populations computed as the fraction of trajectories in the relevant state. The recovery of the $\mathrm{S}_{1}$ state 230 population without state limiting is shown in Figure 4 for both PPV (top panel) and PPE (bottom panel). As can be seen, the relaxation dynamics are independent of the number of states in both model systems. This is, in part, due to the inclusion of the instantaneous decoherence correction which ensures that the adiabatic state populations correctly follow the classically occupied state re235 gardless of the number of states. [25] The other factor is the number of excited states included relative to the number of states that actively participate in the relaxation process. The highest initial state with significant initial population by laser excitation is $\mathrm{S}_{12}$ and $\mathrm{S}_{7}$ for PPV and PPE, respectively. The inclusion of more than 3 higher excited states (corresponding to an excess energy greater

240 than $0.11 \mathrm{eV}$ for PPV and $0.10 \mathrm{eV}$ for PPE) does not affect the relaxation process and the results are independent of the addition of more highest energy excited states.

\subsection{Performance of State Limiting}

Starting from $\mathrm{N}_{S}=15$ for PPV and $\mathrm{N}_{S}=10$ for PPE, we implement the state limiting method using both the state number criteria and the energy-based state limit. For the state number criteria, thresholds of 0 and 2 states $\left(\mathrm{S}_{\alpha+0}\right.$ and $\mathrm{S}_{\alpha+2}$, respectively) were tested such that after each attempted or successful hop, any state that is directly above $\left(\mathrm{S}_{\alpha+0}\right)$ or more than 2 states above $\left(\mathrm{S}_{\alpha+2}\right)$ the current state is removed from the remainder of the dynamics, as shown in Scheme 1. To test the energy-based state limit, we first use a constant energy threshold defined by the total kinetic energy $\left(K E_{\text {total }}\right)$ which represents the upper bound for defining forbidden transitions to higher energy states. In this case, the energy threshold is not redefined at each attempted hop but is rather taken to be the time-averaged total kinetic energy, $\left\langle K E_{\text {total }}\right\rangle$, from the 255 corresponding full simulations without state limiting, giving values of $1.82 \mathrm{eV}$ and $3.36 \mathrm{eV}$ for PPV and PPE, respectively. This is then compared to an 
energy threshold given by the local kinetic energy, $K E_{T D}$ from Eq. 5, which is defined at every attempted hop during dynamics. In Figure 5, the recovery of the $S_{1}$ state population during the relaxation dynamics for the different state limiting procedures are compared to the results from the full simulation with no reduction $\left(\mathrm{N}_{S}=15\right.$ for PPV; $\mathrm{N}_{S}=10$ for PPE). For both systems, the dynamics with state limiting reproduce the dynamics from the full simulation with very good agreement. The only exception being when no additional higher energy states are retained $\left(\mathrm{S}_{\alpha+0}\right)$, indicating that hops to higher energy states are a critical element for FSSH simulations.

The evolution of the average number of states $\left(\mathrm{N}_{S}\right)$ during the dynamics with different state limiting schemes is shown in the top panels of Figure 6 and the relative speed-up compared to the full simulation is provided in Table 1. In both systems, the 2-state limit results in the fastest reduction in the number of states 270 and provides the largest computational savings being 2.6 and 4.3 times faster in PPV and PPE, respectively, compared to the full simulations while still being able to reproduce the correct relaxation dynamics. When the system reaches $\mathrm{S}_{1}$, only $\mathrm{N}_{S}=3$ states are retained. In contrast, the energy-based limit using the total kinetic energy $\left\langle K E_{\text {total }}\right\rangle$ results in the least computational savings and the slowest reduction in the number of states in both systems.

For PPE there is no reduction in the number of states when the energy threshold is defined by the total kinetic energy. This can be understood by tracking the average energies and energy gaps during the full simulation $\left(\mathrm{N}_{S}=15\right.$ for PPV; $\mathrm{N}_{S}=10$ for PPE) plotted in the bottom panels of Figure 6. Here, the average total kinetic energy $\left(K E_{\text {total }}\right)$, the average energy gap between the current state and the state directly above in energy $\left(E_{(\alpha+1)}-E_{\alpha}\right)$, and the average energy gap between the highest energy state and the current state $\left(E_{N_{S}}-E_{\alpha}\right)$ are considered. In both systems, the average energy gap between the current state and the state directly above in energy is much smaller than the total kinetic energy, this explains why the state number criteria results in a faster reduction in the number of states. In order for states to be removed using the energy-based limit, the gap between the highest energy state, $E_{N_{S}}$, and the current state must be larger than the imposed energy threshold. If this is not true, then there are no states above the energy window to be removed.

290 For the case of the total kinetic energy, this means $E_{N_{S}}-E_{\alpha}>K E_{\text {total }}$ must be true for states to be removed, as shown in Scheme 1. In PPV, this criteria is met, on average, around $400 \mathrm{fs}$ out of the 1 ps trajectory duration. However in PPE, $K E_{\text {total }}$ is always much greater than $E_{N_{S}}-E_{\alpha}$, even when the system has relaxed to $\mathrm{S}_{1}$, due to the dense manifold of excited states (See DOES in 295 Figure 3). The usefulness of the total kinetic energy as the threshold for an energy-based state limit depends on the system and its relative electronic energy levels, thus demonstrating that the total kinetic energy is an unreliable and poor choice for this application.

On the other hand, the local kinetic energy performs much better and is not 300 system dependent. In that case $E_{N_{S}}-E_{\alpha}>K E_{T D}$ is true for both systems as seen in the energy plots in the bottom panels of Figure 6. This allows states above $E_{\alpha}+K E_{T D}$ to be removed in both PPV and PPE leading to the reduction 
in the total number of states seen in the top panels of Figure 6 when $\mathrm{KE}_{T D}$ is used as the energy threshold.

\subsection{Excitonic Localization Effects}

The difference between $\mathrm{KE}_{T D}$ and $\mathrm{KE}_{\text {total }}$ is greater in PPE than in PPV. Excitonic states in PPV are delocalized, such that many of the atoms hold transition density and contribute to the local kinetic energy. In contrast, for PPE the localization of the excitation in individual fragments means that there is a greater discrepancy between the number of atoms contributing to the local kinetic energy and the total number of atoms. This can be seen by analyzing the ratio of the participation number to the number of atoms, $\mathrm{P} / \mathrm{N}_{\text {atom }}$. This ratio represents the fraction of atoms represented in the local kinetic energy and will be constrained to $1 / \mathrm{N} \leq \mathrm{P} / \mathrm{N}_{\text {atom }} \leq 1$ where $1 / \mathrm{N}_{\text {atom }}$ represents com315 plete localization and 1 represents complete delocalization. Therefore, $0.025 \leq$ $\mathrm{P} / \mathrm{N}_{\text {atom }} \leq 1$ for PPV and $0.012 \leq \mathrm{P} / \mathrm{N}_{\text {atom }} \leq 1$ for PPE. A histogram of this ratio can be constructed by considering all attempted hopping instances for all of the converged NA-ESMD trajectories and is shown in Figure 7. The average value of $\mathrm{P} / \mathrm{N}_{\text {atom }}$ is $0.33 \pm 0.05$ for PPV and $0.21 \pm 0.03$ for PPE confirming that a smaller fraction of atoms contribute to the local kinetic energy in PPE than in PPV due to stronger localization in PPE.

Similarly, the ratio between the local kinetic energy and the total kinetic energy $\mathrm{KE}_{T D} / \mathrm{KE}_{\text {total }}$ can also be analyzed and is shown in the histogram in Figure 7. In this case, the ratio represents the fraction of kinetic energy con325 tributed by the selected atoms with the largest transition density and has a value of $0.37 \pm 0.07$ for PPV and $0.23 \pm 0.04$ in PPE again confirming the stronger localization in PPE. Although not reflected within the errors associated with the average values, we observe that, in most instances, the fraction of kinetic energy $\left(\mathrm{KE}_{T D} / \mathrm{KE}_{\text {total }}\right)$ is larger than the fraction of total atoms represented $\left(\mathrm{P} / \mathrm{N}_{\text {atom }}\right)$ indicating that the vibrational excitation and transition density localization are concomitant effects.

\section{Conclusions}

We have developed "on the fly" state limiting methods to decrease the computational expense in trajectory surface hopping simulations of realistically large molecular systems with dense manifold of excited states participating in the dynamics. We have implemented these schemes within the NA-ESMD framework and tested the performance on the relaxation dynamics in photoexcited PPV and PPE oligomers. The FSSH algorithm is robust to this kind of treatment, as the dynamics incorporating a state limit method are in agreement with the full simulations except when no additional higher energy states are retained. We have demonstrated that hops to higher energy states are crucial for the FSSH algorithm. Therefore, there is a delicate balance between retaining enough higher energy states to accurately simulate the relaxation dynamics while not including states that are no longer relevant. Due to differences in the density of states and 
localization among the tested systems, we found that the introduction of a local kinetic energy, defined by the contributions from the atoms involved in the localization of the wavefunction, removed any variations in performance that can result from using an arbitrary energy threshold or state cutoff. This provides a factor of 2 in computational saving for the tested systems.

350 The state number criteria is arbitrary and, although it performed well for the tested systems when 2 additional states were retained, it may not work well for other systems as it is unclear exactly what number of states to choose when assigning a state cutoff. For the energy-based state limit, the total kinetic energy proved to be an insufficient energy threshold that depends on the relative value of the kinetic energy compared to the energy spanned by the density of excited states. We found that the use of the local kinetic energy was essential in systems with localized excitations (PPE), since the total kinetic energy and local kinetic energy vary more for systems with localized excitations compared to delocalized systems (PPV). Therefore, the local kinetic energy threshold combined with the energy-based state limit provides a physically relevant approach that can be extended to any molecular system without requiring a priori knowledge of the excited state manifold. Most importantly, the observed reduction of numerical cost would reach orders of magnitude in larger molecular systems with hundreds of excited states.

\section{Acknowledgments}

We thank V. Y. Chernyak for many fruitful discussions that helped to develop the NA-ESMD framework. This research used resources provided by the Los Alamos National Laboratory Institutional Computing Program. We acknowledge support of the U.S. Department of Energy through the Los Alamos 370 National Laboratory (LANL) LDRD Program. LANL is operated by Los Alamos National Security, LLC, for the National Nuclear Security Administration of the U.S. Department of Energy under contract DE-AC52-06NA25396. We also acknowledge support of the Center for Nonlinear Studies (CNLS) and the Center for Integrated Nanotechnology (CINT) at LANL. S.F.A. is supported by CONICET, UNQ, ANPCyT (PICT-2010-2375). 


\section{Tables}

Table 1: Numerical speed-up defined as the ratio (full sim./limited sim.) of average CPU times for different state limiting schemes

\begin{tabular}{|c|cc|}
\hline & PPV3 & $2-3-4$ PPE \\
\hline full simulation & 1.0 & 1.0 \\
0 states $\left(\mathrm{S}_{\alpha+0}\right)$ & 3.5 & 6.3 \\
2 states $\left(\mathrm{S}_{\alpha+2}\right)$ & 2.6 & 4.3 \\
$\left\langle\mathrm{KE}_{\text {total }}\right\rangle$ & 1.2 & 1.0 \\
$\mathrm{KE}_{T D}$ & 2.3 & 1.7 \\
\hline
\end{tabular}




\section{Schemes \& Figures}

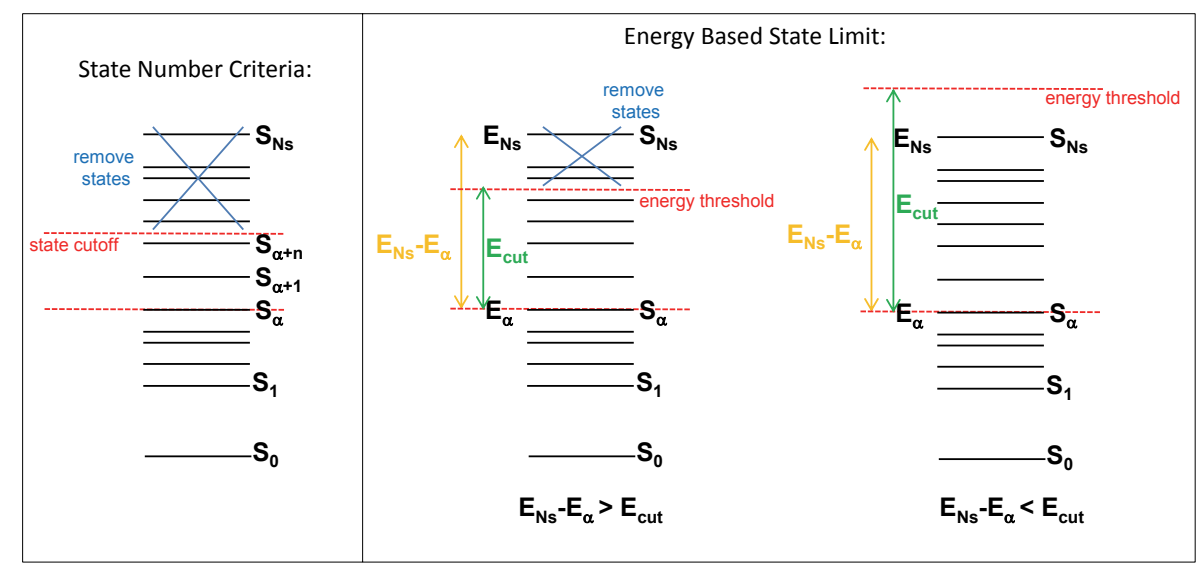

Scheme 1: State number criteria and energy-based state limit showing the current state $\mathrm{S}_{\alpha}$, highest energy state $\mathrm{S}_{N_{S}}$, state cutoff, and energy thresholds. 

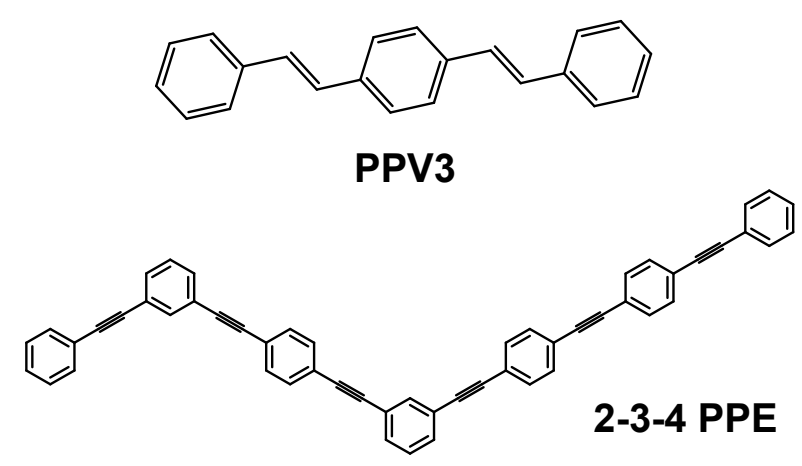

Figure 1: Molecular structure of model systems. A 3 ring oligomer of PPV (PPV3) and meta-substituted 2-,3-, and 4-ring linear PPE segments (2-3-4 PPE) 


\section{PPV3}

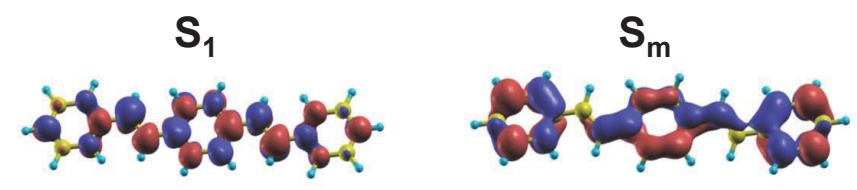

\section{2-3-4 PPE}

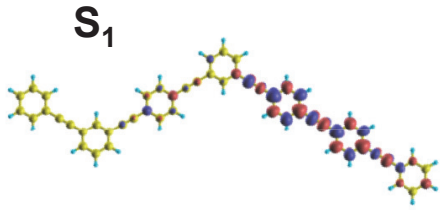

$\mathbf{S}_{3}$

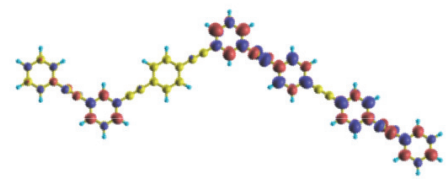

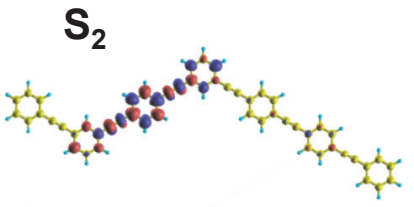

$\mathrm{S}_{4}$

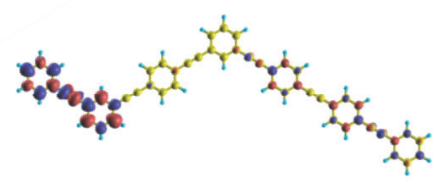

Figure 2: Electronic transition densities for relevant states computed as vertical transitions from the optimized GS structures. (Top) In PPV3, $S_{1}$ and $S_{m}$ are both delocalized excitonic states. (Bottom) In PPE, the four lowest energy excitations are localized on the linear segments. 


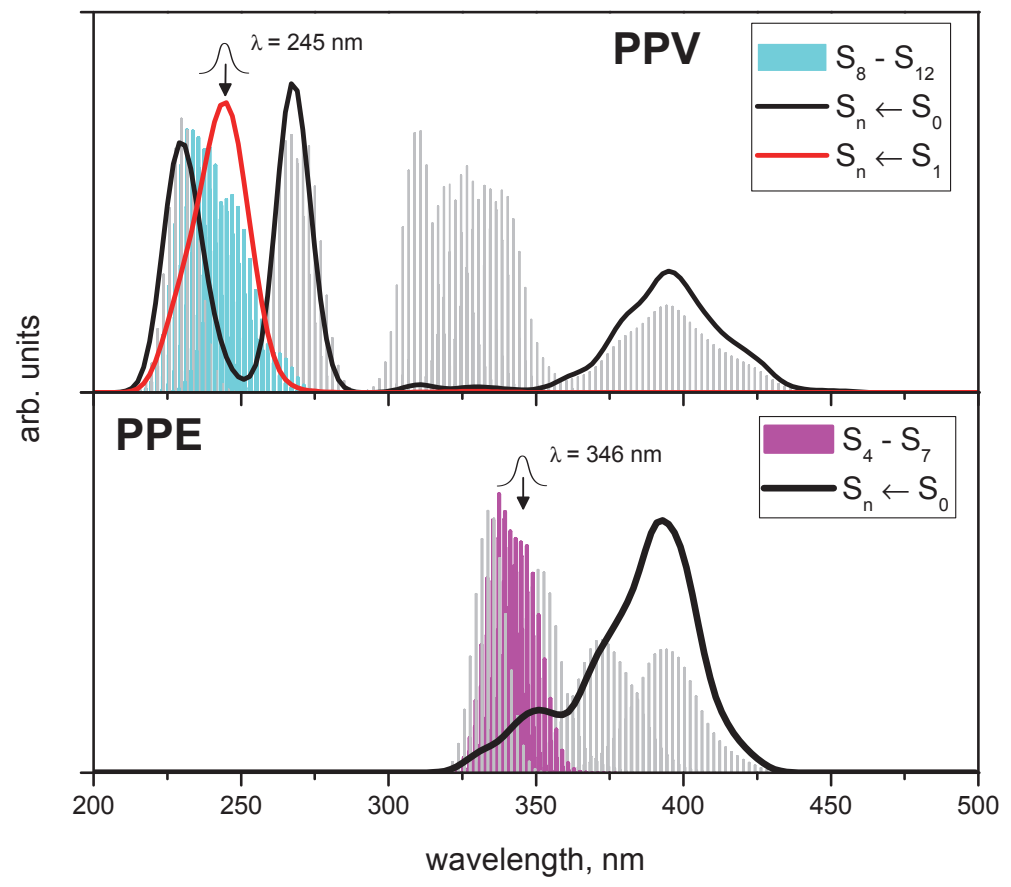

Figure 3: Equilibrated absorption spectra and density of excited states (DOES) for model systems at room temperature $(300 \mathrm{~K})$. (Top) Ground state $\mathrm{S}_{n} \leftarrow \mathrm{S}_{0}$ (black) and lowest excited state $\mathrm{S}_{n} \leftarrow \mathrm{S}_{1}$ (red) absorption for PPV and DOES for the lowest 15 states. $\mathrm{S}_{m}$ corresponds to states $\mathrm{S}_{8}-\mathrm{S}_{12}$ (shown in blue) excited at $\lambda=245 \mathrm{~nm}$. (Bottom) Ground state $\mathrm{S}_{n} \leftarrow \mathrm{S}_{0}$ (black) absorption for PPE and DOES for the lowest 8 excited states. Excitation of the state localized on the 2-ring segment is performed at $\lambda=346 \mathrm{~nm}$ corresponding to states $\mathrm{S}_{4}$ to $\mathrm{S}_{7}$ (shown in magenta). 


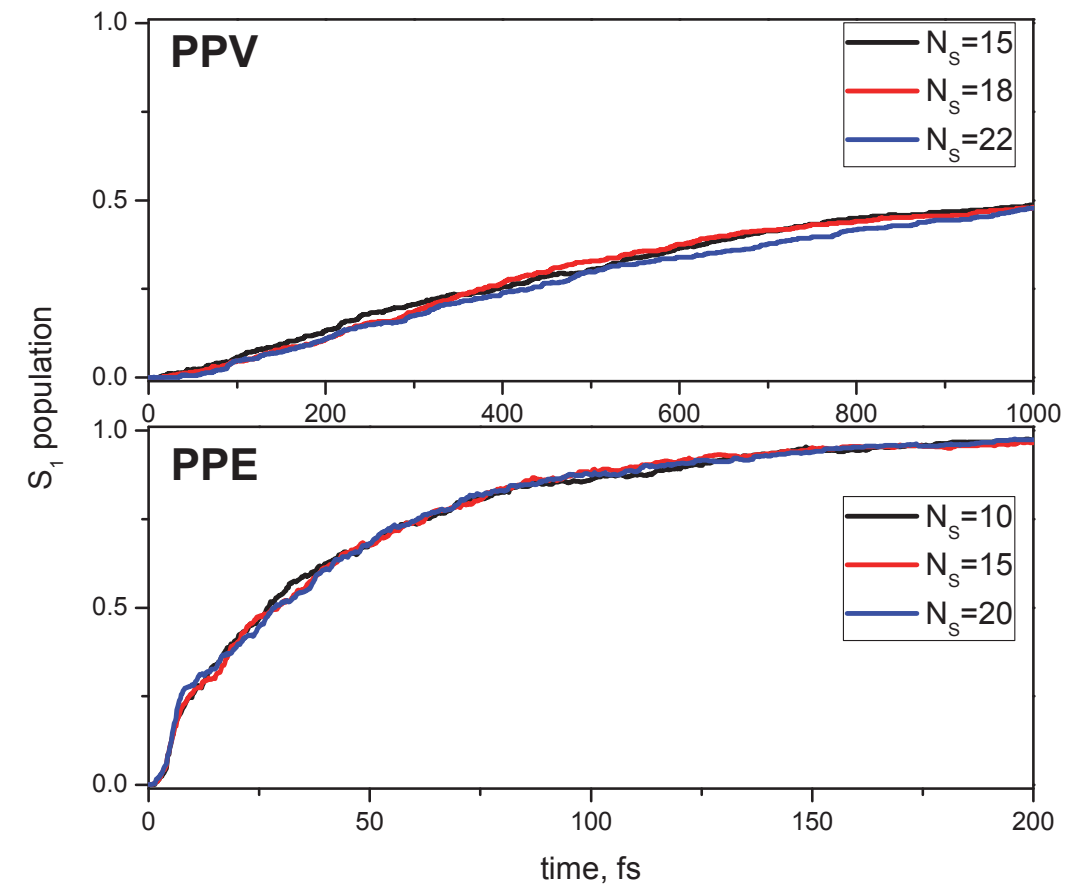

Figure 4: Recovery of the $\mathrm{S}_{1}$ state population during NA-ESMD simulations without state limiting for PPV (Top) and PPE (Bottom). The number of excited states included in the simulation $\mathrm{N}_{S}$ has been varied to show that the relaxation dynamics does not depend on the total number of states. 


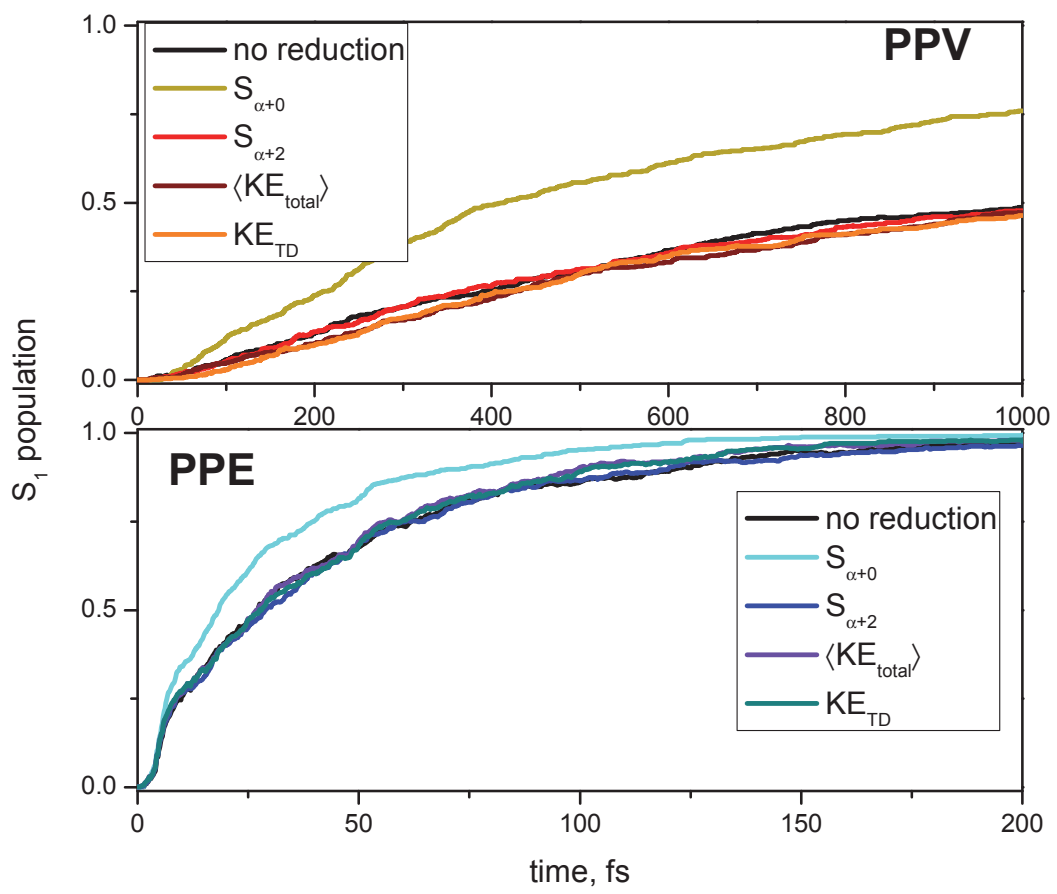

Figure 5: Recovery of the $\mathrm{S}_{1}$ state population during relaxation dynamics with different state limiting schemes compared to the full simulation without state limiting for PPV (Top) and PPE (Bottom). 


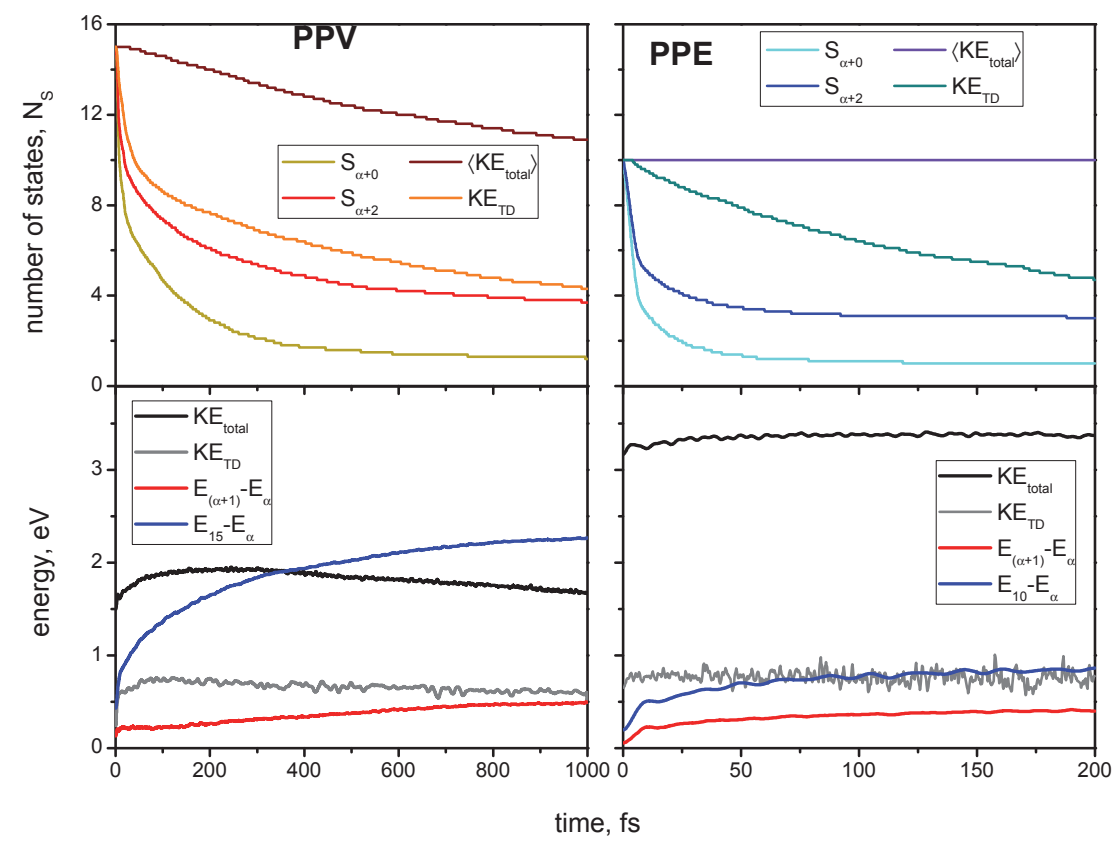

Figure 6: (Top)The evolution of the average number of states $\left(\mathrm{N}_{S}\right)$ during the dynamics with different state limiting schemes. (Bottom) The evolution of the relevant energies and energy gaps averaged over all trajectories. The total kinetic energy $\left(\mathrm{KE}_{\text {total }}\right.$; black), the local kinetic energy based on transition density analysis $\left(\mathrm{KE}_{T D}\right.$; grey), the energy gap between the current state and the state directly above in energy $\left(\mathrm{E}_{(\alpha+1)}-\mathrm{E}_{\alpha}\right.$; red), and the energy gap between the highest energy state and the current state $\left(\mathrm{E}_{N_{S}}-\mathrm{E}_{\alpha} ;\right.$ blue) are plotted for PPV (Left) and PPE (Right). 


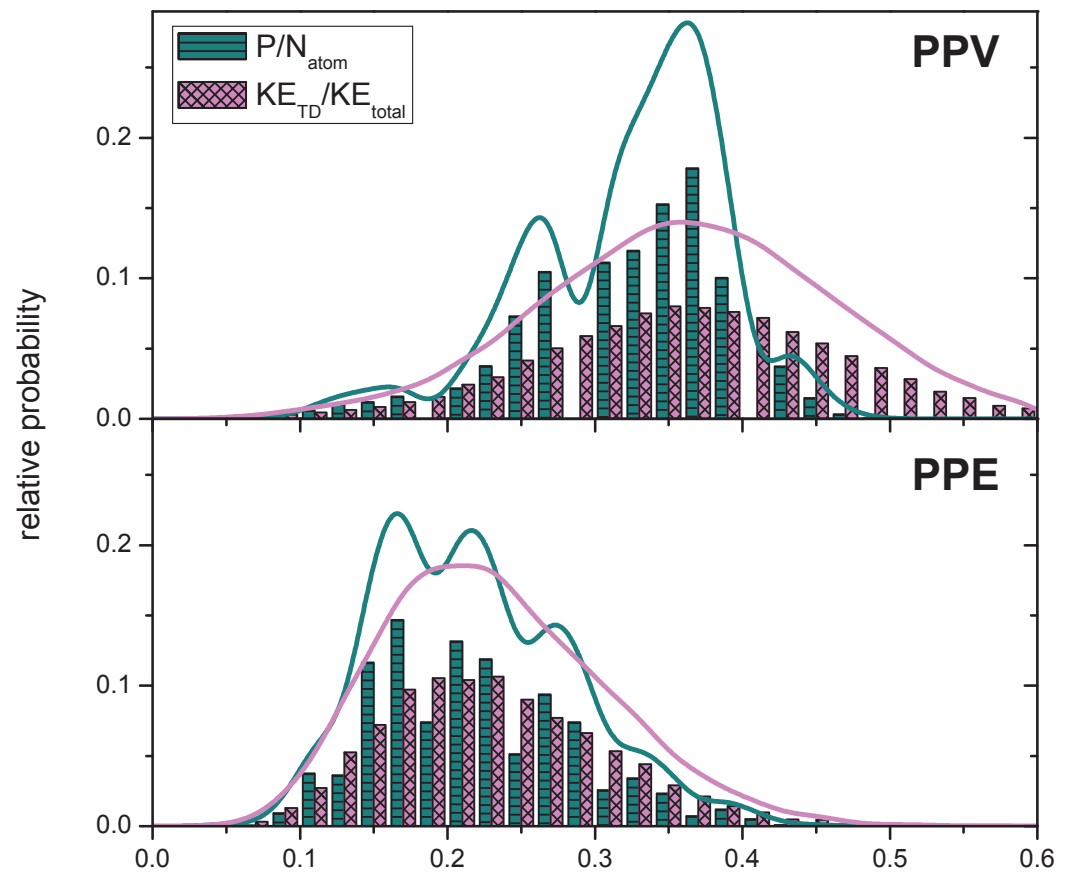

Figure 7: Histograms of the relevant ratios computed at all attempted hopping times for PPV (Top) and PPE (Bottom). The ratio of participation number per atom to total number of atoms $\left(\mathrm{P}_{\text {atom }} / \mathrm{N}_{\text {atom }}\right)$ and the ratio of the contribution to the kinetic energy from the atoms carrying the transition density to the total kinetic energy $\left(\mathrm{KE}_{T D} / \mathrm{KE}_{\text {total }}\right)$ are shown. 


\section{References}

[1] J. Tully, Molecular dynamics with electronic transitions, J. Chem. Phys. 93 (2) (1990) 1061-1071.

[2] F. Plaser, G. Granucci, J. Pittner, M. Barbatti, M. Persico, H. Lischka, Surface hopping dynamics using a locally diabatic formalism: Charge transfer in the ethylene dimer cation and excited state dynamics in the 2-pyridone dimer, J. Chem. Phys. 137 (2012) 22A514.

[3] A. V. Akimov, A. J. Neukirch, O. V. Prezhdo, Theoretical insights into photoinduced charge transfer and catalysis at oxide interfaces, Chem. Rev. 113 (2013) 4496-4565.

[4] L. A. Hernandez, T. Nelson, S. Tretiak, S. Fernandez-Alberti, Photoexcited energy transfer in a weakly coupled dimer, J. Phys. Chem. B 119 (2015) $7242-7252$.

[5] S. Fernandez-Alberti, A. Roitberg, V. Kleiman, T. Nelson, S. Tretiak, Shishiodoshi unidirectional energy transfer mechanism in phenylene ethynylene dendrimers, J. Chem. Phys. 137 (2012) 22A526.

[6] T. Nelson, S. Fernandez-Alberti, A. Roitberg, S. Tretiak, Conformational disorder in energy transfer: Beyond forster theory, Phys. Chem. Chem. Phys. 15 (2013) 9245-9256.

[7] S. Hammes-Schiffer, J. C. Tully, Proton-transfer in solution - moleculardynamics with quantum transitions, J. Chem. Phys. 101 (6) (1994) 46574667.

[8] J.-Y. Fang, S. Hammes-Schiffer, Comparison of surface hopping and mean field approaches for model proton transfer reactions, J. Chem. Phys. 110 (1999) 11166.

[9] S.-H. Xia, B.-B. Xie, Q. Fang, G. Cui, W. Thiel, Excited-state intramolecular proton transfer to carbon atoms: Nonadiabatic surface-hopping dynamics simulations, Phys. Chem. Chem. Phys. 17 (2015) 9687-9697.

[10] K. Rajak, B. Maiti, Trajectory surface hopping study of the $\mathrm{o}\left({ }^{3} \mathrm{p}\right)+\mathrm{c}_{2} \mathrm{~h}_{2}$ reaction dynamics: Effect of collision energy on the extent of intersystem crossing, J. Chem. Phys. 140 (2014) 044314.

[11] N. Balucani, F. Leonori, O. Casavecchia, B. Fu, J. M. Bowman, Crossed molecular beams and quasiclassical trajectory surface hopping studies of the multichannel nonadiabatic $\mathrm{o}\left({ }^{3} \mathrm{p}\right)+$ ethylene reaction at high collision energy, J. Phys. Chem. A 119 (2015) 12498-12511.

[12] M. Boggio-Pasqua, C. F. Burmeister, M. A. Robb, G. Groenhof, Photochemical reactions in biological systems: Probing the effect of the environment by means of hybrid quantum chemistry/molecular mechanics simulations, Phys. Chem. Chem. Phys. 14 (2012) 7912-7928. 
[13] L. Yue, Z. Lan, Y.-J. Liu, The theoretical estimation of the bioluminescent efficiency of the firefly via a nonadiabatic molecular dynamics simulation, J. Phys. Chem. Lett. 6 (2015) 540-548.

[14] T. Nelson, S. Fernandez-Alberti, V. Chernyak, A. E. Roitberg, S. Tretiak, Nonadiabatic excited-state molecular dynamics modeling of photoinduced dynamics in conjugated molecules, J. Phys. Chem. B 115 (2011) 5402-5414.

[15] T. Nelson, S. Fernandez-Alberti, A. E. Roitberg, S. Tretiak, Nonadiabatic excited-state molecular dynamics: Modeling photophysics in organic conjugated materials, Acc. Chem. Res. 47 (2014) 1155-1164.

[16] D. J. Thouless, The Quantum Mechanics Of Many-Body Systems, Academic Press, New York, 1972.

[17] F. Furche, On the density matrix based approach to time-dependent density functional response theory, J. Chem. Phys. 114 (14) (2001) 5982-5992.

[18] F. Furche, R. Ahlrichs, Adiabatic time-dependent density functional methods for excited state properties, J. Chem. Phys. 117 (16) (2002) 7433-7447.

[19] S. Tretiak, V. Chernyak, Resonant nonlinear polarizabilities in the timedependent density functional (tddft) theory, J. Chem. Phys. 119 (17) (2003) 8809-8823.

[20] V. Chernyak, S. Mukamel, Density-matrix representation of nonadiabatic couplings in time-dependent density functional (tddft) theories, J. Chem. Phys. 112 (8) (2000) 3572-3579.

[21] M. Tommasini, V. Chernyak, S. Mukamel, Electronic density-matrix algorithm for nonadiabatic couplings in molecular dynamics simulations, Int. J. Quantum Chem. 85 (4-5) (2001) 225-238.

[22] J. Pittner, H. Lischka, M. Barbatti, Optimization of mixed quantumclassical dynamics: Time-derivative coupling terms and selected couplings, Chem. Phys. 356 (2007) 147-152.

[23] M. Barbatti, H. Lischka, Nonadiabatic deactivation of 9h-adenine: A comprehensive picture based on mixed quantum-classical dynamics, J. Am. Chem. Soc. 130 (21) (2008) 6831-6839.

[24] T. Nelson, S. Fernandez-Alberti, V. Chernyak, A. Roitberg, S. Tretiak, Nonadiabatic excited-state molecular dynamics: Numerical tests of convergence and parameters, J. Chem. Phys. 136 (2012) 054108.

[25] T. Nelson, S. Fernandez-Alberti, A. E. Roitberg, S. Tretiak, Nonadiabatic excited-state molecular dynamics: Treatment of electronic decoherence, J. Chem. Phys. 138 (2013) 224111. 
[26] M. Soler, A. Roitberg, T. Nelson, S. Tretiak, S. Fernandez-Alberti, Analysis of state-specific vibrations coupled to the unidirectional energy transfer in conjugated dendrimers, J. Phys. Chem. A 116 (2012) 9802.

[27] P. M. Shenai, S. Fernandez-Alberti, W. P. Bricker, S. Tretiak, Y. Zhao, Internal conversion and vibrational energy redistribution in chlorophyll a, J. Phys. Chem. B 120 (2016) 49-58.

[28] S. Fernandez-Alberti, A. Roitberg, T. Nelson, S. Tretiak, Identification of unavoided crossings in nonadiabatic photoexcited dynamics involving multiple electronic states in polyatomic conjugated molecules, J. Chem. Phys. 137 (2012) 014512.

[29] T. Nelson, S. Fernandez-Alberti, A. E. Roitberg, S. Tretiak, Artifacts due to trivial unavoided crossings in the modeling of photoinduced energy transfer dynamics in extended conjugated molecules, Chem. Phys. Lett 590 (2013) 208-213.

[30] M. A. Soler, T. Nelson, A. E. Roitberg, S. Tretiak, S. Fernandez-Alberti, Signature of nonadiabatic coupling in excited-state vibrational modes, J. Phys. Chem. A 118 (2014) 10372-10379.

[31] J. A. Bjorgaard, T. Nelson, K. Kalinin, V. Kuzmenko, K. A. Velizhanin, S. Tretiak, Simulations of fluorescence solvatochromism in substituted ppv oligomers from excited state molecular dynamics with implicit solvent, Chem. Phys. Lett. 631 (2015) 66-69.

[32] S. Tretiak, S. Mukamel, Density matrix analysis and simulation of electronic excitations in conjugated and aggregated molecules, Chem. Rev. 102 (9) (2002) 3171-3212.

[33] S. Mukamel, S. Tretiak, T. Wagersreiter, V. Chernyak, Electronic coherence and collective optical excitations of conjugated molecules, Science 277 (5327) (1997) 781-787.

[34] M. J. S. Dewar, E. G. Zoebisch, E. F. Healy, J. J. P. Stewart, Am1: A new general purpose quantum mechanical molecular model, J. Am. Chem. Soc. 107 (13) (1985) 3902-3909.

[35] J.-Y. Fang, S. Hammes-Schiffer, Improvement of the internal consistency in trajectory surface hopping, J. Phys. Chem. A 103 (1999) 9399-9407.

[36] M. Thachuk, M. Y. Ivanov, D. M. Wardlaw, A semiclassical approach to intense-field above-threshold dissociation in the long wavelength limit. ii. conservation principles and coherence in surface hopping, J. Chem. Phys. 109 (14) (1998) 5747-5760.

[37] M. Paterlini, D. Ferguson, Constant temperature simulations using the langevin equation with velocity verlet integration, Chem. Phys. 236 (1-3) (1998) 243-252. 
[38] S. V. Frolov, Z. Bao, M. Wohlgenannt, Z. V. Vardeny, Ultrafast spectroscopy of even-parity states in pi-conjugated polymers, Phys. Rev. Lett. 85 (10) (2000) 2196-2199.

495 [39] M. Chandross, S. Mazumdar, Coulomb interactions and linear, nonlinear, and triplet absorption in poly(para-phenylvinylene), Phys. Rev. B 55 (3) (1997) 1497-1504. 